\title{
Les hybrides et les haploïdes doublés
}

\section{Hybrids and double haploids}

Oléagineux, Corps Gras, Lipides. Volume 7, Numéro 1, 20-1, Janvier - Février 2000, Filière

Auteur(s) : Jacqueline PIERRE, INRA, UMR Biologie des organismes et des populations appliquée à la protection des plantes [BiO3P], Domaine de la Motte, BP 35327, 35653 Le Rheu Cedex, France.

Résumé : Le thème des hybrides, abordé soit au niveau biotechnologique soit au niveau de la sélection, a fait l'objet de quatorze communications orales et d'une vingtaine de panneaux. Celui des haploïdes doublés a été beaucoup moins développé (une communication, un panneau).

\section{ARTICLE}

Le thème des hybrides, abordé soit au niveau biotechnologique soit au niveau de la sélection, a fait l'objet de quatorze communications orales et d'une vingtaine de panneaux. Celui des haploïdes doublés a été beaucoup moins développé (une communication, un panneau).

La communication la plus synthétique sur les hybrides a été présentée par I.D. Pandey et al. Bien que limitée à la recherche indienne, elle a eu le mérite de dresser une large revue bibliographique (une centaine de références) sur les hybrides chez les Brassica en général (colza, moutarde brune, navettes v. sarson et toria). Cette analyse a été faite selon les points suivants : I'hétérosis, la capacité à la combinaison et le rôle des gènes, l'allofécondation, les outils permettant l'obtention d'hybrides (diverses stérilités mâles, restauration de la fertilité, auto-incompatibilité, gamétocides), les applications à la biotechnologie (recombinaison mitochondriale par fusion de protoplasme, hybridation somatique, analyse des bases moléculaires de la CMS, recherche de diversité génétique par marqueurs, culture d'embryons) et enfin les implications commerciales. Les auteurs ont conclu en soulignant la nécessité de poursuivre des recherches fondamentales dans ces domaines et en rappelant que le devenir des hybrides dépend du rapport bénéfice/coût qu'ils offriront. Les autres interventions et panneaux ont été beaucoup plus spécifiques et ont repris les divers points qui viennent d'être cités.

Ainsi, pour ce qui est de l'effet d'hétérosis les résultats présentés sont divergents selon le type d'hybride et selon que l'on considère l'hétérosis sensu stricto, c'est-à-dire par rapport à la valeur des géniteurs, ou par rapport à celle de variétés classiques. Les données expérimentales indiennes ont montré un net gain de rendement des hybrides par rapport aux variétés commerciales, non seulement chez le colza (10 à $34 \%$ pour des hybrides restaurés Seedlink ${ }^{\mathrm{TM}}$; GoshDastidar et al.) ou la navette v. sarson (21 à $27 \%$ ) mais surtout chez la moutarde brune (33,5\% ; Pandey et Zerh). En Allemagne, les gains de rendement pour des hybrides de colza obtenus selon le système MS Lembke ont été de l'ordre de 5 à $15 \%$ et devraient encore progresser (Frauen et Paulmann). En revanche, les résultats sur les rendements des hybrides en France (Pinochet et Bertrand; Champolivier et Merrien) sont moins optimistes et les expérimentateurs mettent en avant les conditions environnementales 
comme un des facteurs limitant l'allopollinisation et, par conséquent, l'avantage des hybrides. Les problèmes de l'évaluation des hybrides et du risque de la surestimation de leurs rendements dans les essais en vue d'une inscription ont par ailleurs été soulevés (Ramsbotton ; Schott). Cependant, les résultats d'études portant sur la recherche, assistée par marqueurs, de géniteurs présentant une distance génétique importante et une bonne capacité à la recombinaison laissent espérer une maximisation de l'effet d'hétérosis dans les programmes à venir (Girke et al. ; Förster et al.).

Les informations relatives aux systèmes de stérilité mâle ont été très diverses et fréquemment associées à des travaux portant sur la restauration de la fertilité. Si peu d'études sur la stérilité mâle génique (GMS) proprement dite ont été rapportées (GMS janpol ; Bartowiak-Broda et al.), en revanche une équipe chinoise a présenté plusieurs panneaux sur les interactions entre les deux systèmes CMS et GMS (Chen et al. ; Tang et al.). Concernant la stérilité mâle cytoplasmique (CMS), il a été fait état de la poursuite de recherches sur le système lyr (Enarthrocarpus lyratus) qui permet une bonne restauration (Banga et Banga). Pour le système mur, on a trouvé des lignées mainteneuses et restauratrices (McVetty et al.) et pour le système polima il a été démontré qu'il pourrait servir de restaurateur (une paire de gènes dominants impliqués) pour des lignées mâles stériles thermodépendantes (Yang et al.). Les études les plus innovantes ont porté sur l'utilisation de la transgénèse soit pour induire la stérilité mâle grâce à un transgène détruisant les cellules du tapetum en présence de $\mathrm{N}$ acétyl-phosphinithricine (Köhne et al.), technique qui offre l'avantage de ne pas poser de problème de restauration, soit pour contrôler la stérilité grâce à une construction antisens du gène $B c p 1$, spécifique des anthères, transférée par Agrobacterium tumefaciens (Bhalla et Singh). L'exploitation de l'auto-incompatibilité (4 allèles récessifs S) pour aboutir à des croisements a également été évoquée (Möhring et al. ; Kucera et al.). Globalement, il est apparu que la restauration de la fertilité reste un objectif constant quelle qu'en soit la source (localisation d'un gène restaurateur du système CMS tour ; Trendelkamp et al.). Des travaux conduits depuis plusieurs années dans ce sens ont permis l'obtention d'hybrides restaurés chez la moutarde brune en utilisant le système Seedlink TM ou des haploïdes doublés (GoshDastidar et al.). Chez le colza, les premiers hybrides restaurés double zéro (système CMS ogu-INRA) ont été créés par une équipe française (Delourme et al.).

\section{Création de matériel nouveau du point de vue du développement de la plante}

Cette rubrique a trait à la sélection de colzas mutants possédant des caractéristiques morphologiques particulières afin de répondre à des besoins différents. Ainsi trois types de colzas ont été présentés : demi-nains, cléistogames et sans pétales mâles fertiles ou mâles stériles, et leurs intérêts respectifs ont été discutés (Hogarth et Mendham ; Pierre et Renard). *

\section{ACTUALITÉ}

Ayant fait valoir ses droits à la retraite, François Mordret a quitté ses fonctions de Directeur général de l'Iterg au 31 décembre 1999.

Actif collaborateur de la revue, le comité de rédaction lui transmet ses très sincères salutations non sans espérer bénéficier toujours de ses précieux conseils dans le domaine analytique.

Ancien Directeur marketing de Diester Industrie, Frédéric Staat a été nommé Directeur Général de I'Iterg au ${ }^{\text {er }}$ janvier 2000. II rejoint également l'équipe de la rédaction de la revue. 